\title{
Low Frequency Nuclear Quadrupole Resonance with SQUID Amplifiers*
}

\author{
John Clarke \\ Department of Physics, University of California, Berkeley, CA 94720 and \\ Materials Sciences Division, Lawrence Berkeley Laboratory, Berkeley, CA 94720
}

Z. Naturforsch. 49a, 5-13 (1994); received October 18, 1993

\begin{abstract}
The dc SQUID (Superconducting QUantum Interference Device) can be configured as an amplifier of spin-echos with a noise temperature of approximately $10 \mathrm{mK}(\mathrm{f} / 1 \mathrm{MHz})$ at an operating temperature of $1.5 \mathrm{~K}$. A Fourier transform spectrometer based on a SQUID with a superconducting input circuit and operated in a flux-locked loop is used to obtain nuclear quadrupole resonance (NQR) spectra in a broadband mode over the bandwith $0-1 \mathrm{MHz}$. Spin-echo spectra of ${ }^{14} \mathrm{~N}$ in $\mathrm{NH}_{4} \mathrm{ClO}_{4}$ reveal sharp NQR resonances, obtained simultaneously, at $17.4,38.8$ and $56.2 \mathrm{kHz}$. At $1.5 \mathrm{~K}$, the measured longitudinal and transverse relaxation times $T_{1}$ and $T_{2}$ for the $38.8 \mathrm{kHz}$ transition are $63 \pm 3 \mathrm{~ms}$ and $22 \pm 2 \mathrm{~ms}$, respectively.
\end{abstract}

Key words: SQUID, Fourier transform spectrometer, $\mathrm{NQR}, \mathrm{NH}_{4} \mathrm{ClO}_{4}$, spin echo.

\section{Introduction}

Nuclear magnetic resonance (NMR) spectra enable one to measure nuclear magnetic dipole-dipole and electric quadrupole interactions in crystalline solids and thus yield valuable information on the interatomic distances and local electric field gradients [1-3]. However, in polycrystalline or disordered materials the random orientations of molecules with respect to the applied magnetic field produce a "powder pattern" in which structural information may well be obscured. One can overcome this problem by removing the external magnetic field, which defines a direction in space, and performing experiments such as nuclear quadrupole resonance (NQR) [4] or zero field NMR [5], in which all equivalent sites have the same resonant frequencies and the resulting spectra should therefore be sharp. Examples of NQR are ${ }^{2} \mathrm{D}$ in selectively deuterated organic molecules and ${ }^{9} \mathrm{Be},{ }^{11} \mathrm{~B}$ or ${ }^{14} \mathrm{~N}$ in nearly symmetric environments; an example of zero field NMR is water in hydrated crystals. However, the corresponding resonant frequencies $\omega / 2 \pi$ are often very low compared with those in conventional NMR or NQR, for example, about $42 \mathrm{kHz}$ for the zero field NMR of water in hydrated crystals or a few tens of kilohertz for the NQR of ${ }^{14} \mathrm{~N}$ in the ammonium ion. As a result, the signal delivered to a coil

\footnotetext{
* Presented at the XIIth International Symposium on Nuclear Quadrupole Resonance, Zürich, July 19-23, 1993.
}

Reprint requests to John Clarke, Physics Department, University of California, Berkeley, California 94720, U.S.A. surrounding a collection of precessing spins is greatly reduced compared to that at frequencies of, say, tens of megahertz: at a given temperature $T$ the magnetization $\mathbf{M}$ scales as $\omega / T$ and the voltage as $\omega \mathbf{M}$, so that the induced voltage scales as $\omega^{2}$. This small signal implies that it is difficult to observe low-frequency NMR or NQR with a conventional semiconductor amplifier coupled to a resonant circuit, and alternative techniques have therefore been developed. One method is magnetic field cycling [6], which produces the free induction decay (FID) signal point and is thus rather time consuming. In another technique, applicable to zero field NMR, one spins the sample in a high magnetic field and applies radio frequency pulse sequences [7]. A third approach involves a Robinson oscillator [8] in a continuous wave (CW) NQR spectrometer, and has been used successfully at frequencies down to $200 \mathrm{kHz}$ [9].

Yet another method is to detect the low-frequency signal directly by means of an amplifier based on a dc Superconducting QUantum Interference Device (SQUID) [10]. The SQUID can be used in either a CW [11] or pulsed mode [12-14]. At kilohertz frequencies, the SQUID amplifier, as we shall see in Sect. 2, has a noise temperature far below that of conventional amplifiers. The SQUID offers a unique feature in that it can be used to detect magnetic flux, rather than the rate of change of magnetic flux, so that at a given temperature the detected signal scales with $\omega$, rather than as $\omega^{2}$. Furthermore, in this mode the input circuit is nonresonant so that one can measure resonance signals over a broad bandwidth, in the present case

0932-0784 / 94 / 0100-0005 \$01.30/0. - Please order a reprint rather than making your own copy. 
from zero to about $1 \mathrm{MHz}$ [15]. The direct detection of the precessing spins enables one to take advantage of the rich variety of pulse techniques used in higher frequency NMR and NQR measurements.

Section 2 provides an overview of the SQUID and its operation as an amplifier. Section 3 describes the NQR spectrometer and Sect. 4 presents the results of its use to detect NQR in $\mathrm{NH}_{4} \mathrm{ClO}_{3}$; these sections are based on [12] and [13], respectively. Section 5 contains some concluding remarks.

\section{DC SQUIDs}

\subsection{Principle of operation}

The dc SQUID combines two superconducting phenomena: flux quantization and Josephson tunneling. Flux quantization demands that the net magnetic flux threading a closed superconducting loop be quantized in units of the flux quantum, $\Phi_{0}=h /$ $2 e \approx 2.07 \times 10^{-15} \mathrm{~Wb}$, where $h$ is Planck's constant and $2 e$ is the charge on a Cooper pair. Thus, if we cool a ring through its superconducting transition temperature in the presence of a magnetic field and then remove the field, a persistent supercurrent flowing around the ring will maintain a flux $n \Phi_{0}$, where $n$ is an integer. If we then apply an external magnetic flux to the ring, the supercurrent will adjust itself to ensure that the net flux remains at $n \Phi_{0}$. The Josephson tunnel junction consists of two superconductors separated by a thin barrier. Cooper pairs are able to tunnel coherently through the barrier, enabling the junction to support a supercurrent up to a maximum value known as the critical current $I_{0}$; this zero voltage current constitutes the dc Josephson effect. When the applied current exceeds $I_{0}$, a voltage $V$ is developed across the junction. In this regime, the supercurrents oscillate at a frequency $2 e V / h=V / \Phi_{0}$; this is the ac Josephson effect.

The dc SQUID, shown in Fig. $1 \mathrm{a}$, consists of two Josephson junctions connected in parallel on a superconducting loop of inductance $L$. Each tunnel junction has associated with it a capacitance $C$, and is shunted with an external resistance $R$ to produce the nonhysteretic current-voltage $(\mathrm{I}-\mathrm{V})$ characteristic shown in Fig. 1 (b) [16]. When we change the magnetic flux applied to the loop, the critical current oscillates between a maximum value at $\Phi=n \Phi_{0}$ and a minimum value at $\Phi=(n+1 / 2) \Phi_{0}$; thus, the period of oscillation is $\Phi_{0}$. If we bias the SQUID with an appropriate bias current $I_{\mathrm{B}}$, the voltage correspondingly oscillates be-

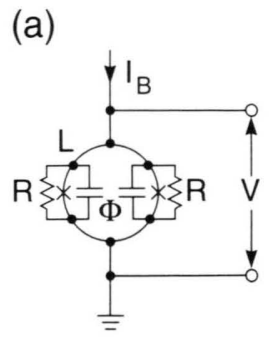

(b)

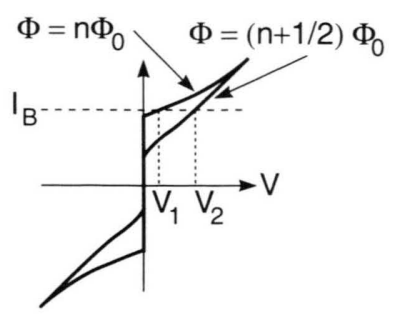

(c)

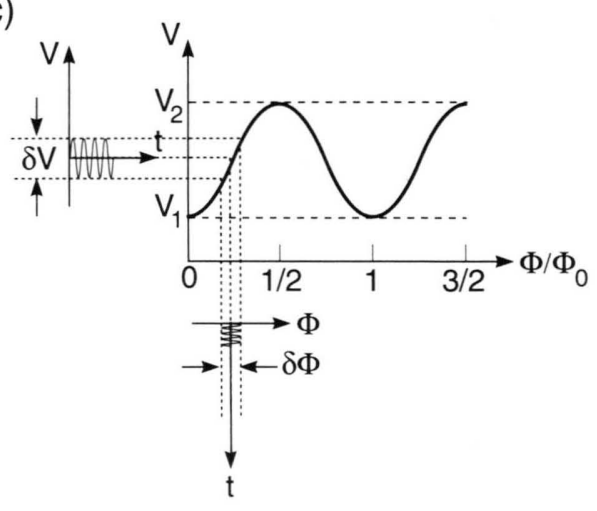

Fig. 1. (a) Configuration of dc SQUID; (b) current-voltage (I-V) characteristics for an applied flux $\Phi$ of $n \Phi_{0},(n+1 /$ 2) $\Phi_{0}$; (c) $V$ vs. $\Phi$ for constant bias current $I_{\mathrm{B}}$, indicating how flux $\delta \Phi$ is converted into voltage $\delta V$.

tween $V_{1}$ and $V_{2}$, as indicated in Figs. $1 \mathrm{~b}$ and c. In practice, one uses a steep portion of the $V-\Phi$ curve (Fig. $1 \mathrm{c}$ ) to convert an oscillating flux into an oscillating voltage that can be amplified with conventional electronics. Thus, in essence, the SQUID is a flux-tovoltage transducer with a transfer coefficient $V_{\Phi}=(\partial V)$ $\partial \Phi)_{I}$. Computer simulations for an optimized SQUID yield

$$
V_{\Phi} \approx R / L
$$

\subsection{Practical devices}

Modern SQUIDs are made from thin films deposited on silicon wafers and patterned with photolithographic and etching techniques taken over from the semiconductor industry. As many as 400 SQUIDs can be produced on a 4-inch wafer; subsequently, the wafer is diced into chips, each with one SQUID. The configuration of the SQUID (Fig. 2) generally follows that first introduced by Ketchen and Jaycox [17]. The body of the SQUID consists of a square washer of niobium with a narrow radial slit. A Josephson junction is grown on each side of the outside end of this , 


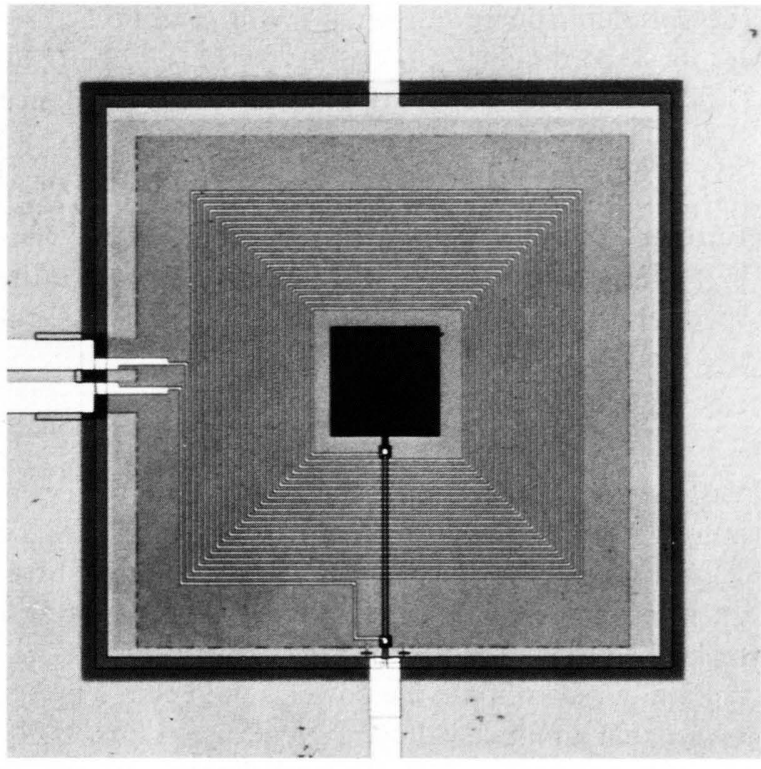

(2a)

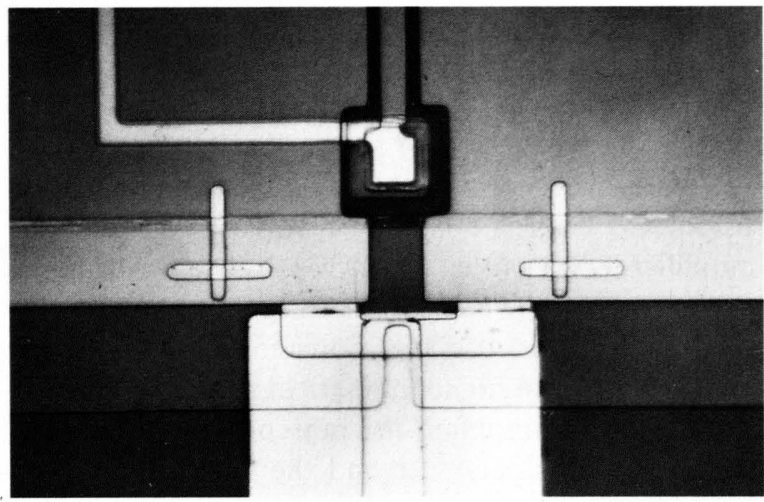

(2b)

Fig. 2. (a) Photograph of thin-film dc SQUID. The square niobium washer is about $1 \mathrm{~mm}$ across. A slit in the washer runs from the central square hole to the outer edge. There is a Josephson tunnel junction on each side of the slit near the outer edge, as shown in the enlarged view of this region, see b. Each junction is defined by two crossed slits, about $2 \mu \mathrm{m}$ wide, in an insulating film deposited on the niobium washer. The junctions are completed, and connected together, by an upper superconducting film. When a current source is connected to the two central leads shown in (a) a current flows between the washer and the upper film via the two Josephson junctions. The voltage is measured across the same two leads. The two leads at the left in (a) provide contact between the pick-up coil shown in Fig. 7 and the 20-turn input coil, the innermost turn of which is contacted via a narrow film running in the slit of the washer. (Courtesy Non Fan and M. B. Heaney). slit, and the upper electrodes of these junctions are connected by a second superconducting layer, insulated from the first, to complete the loop. Signals are coupled into the SQUID via a multiturn, spiral coil, also made of niobium, which is deposited on an insulating layer covering the square washer. Virtually all the flux produced by a current in this coil is forced to flow through the central hole in the washer, giving very efficient coupling.

In almost all applications, the SQUID is operated in a flux-locked loop (see Sect. 3). A voltage induced across the SQUID by a flux change is amplified and fed, via a resistor, into a second coil inductively coupled to the SQUID. The feedback flux opposes the applied flux, so that the SQUID is a null detector; the voltage across the feedback resistor is proportional to the flux signal. This configuration effectively linearizes the SQUID response, enabling one to detect changes in flux corresponding either to many flux quanta or to a very small fraction of one flux quantum. At frequencies $f$ above the $1 / f$ noise knee (typically $1 \mathrm{~Hz}$ ), the spectral density $S_{\Phi}(f)$ of the flux noise is white; for a SQUID operating in liquid helium at $4.2 \mathrm{~K} S_{\Phi}^{1 / 2}(f)$ is order of the $10^{-6} \Phi_{0} \mathrm{~Hz}^{-1 / 2}$. It is useful to express this flux noise as a noise energy, $S_{\Phi}(f) / 2 L$, which is of the order of $10^{-32} \mathrm{~J} \mathrm{~Hz}^{-1}$ for a loop inductance $L \sim$ $100 \mathrm{pH}$. It is this extraordinary energy sensitivity that enables one to measure low-frequency NQR and NMR signals.

\subsection{Noise temperature and gain}

We turn now to a brief discussion of the noise temperature and gain of a dc SQUID configured as an amplifier. Figure $3 \mathrm{a}$ shows the two white noise sources of the SQUID: a voltage noise $V_{\mathrm{N}}(t)$ across the device and a current noise $J_{\mathrm{N}}(t)$ around the SQUID loop. Both noise terms originate in the Nyquist noise of the two shunting resistors, $R$, each of which has a current spectral density $4 k_{\mathrm{B}} T / R$, where $k_{\mathrm{B}}$ is Boltzmann's constant. The spectral densities of $V_{\mathrm{N}}(t)$ and $J_{\mathrm{N}}(t)$ for an optimized SQUID have been computed to be

$$
S_{\mathrm{V}}(f) \approx 16 k_{\mathrm{B}} T R
$$

and

$$
S_{\mathrm{J}}(f) \approx 11 k_{\mathrm{B}} T / R,
$$

respectively $[18,19]$. In addition, $V_{\mathrm{N}}(t)$ and $J_{\mathrm{N}}(t)$ are partially correlated, with a cross-spectral density

$$
S_{\mathrm{VJ}}(f) \approx 12 k_{\mathrm{B}} T \text {. }
$$


(a)

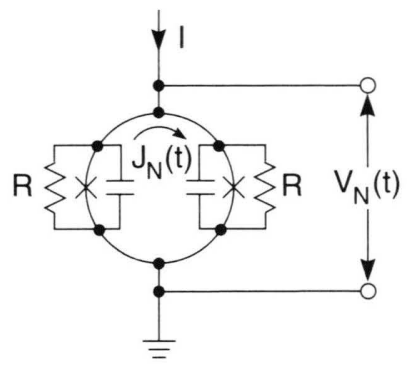

(b)

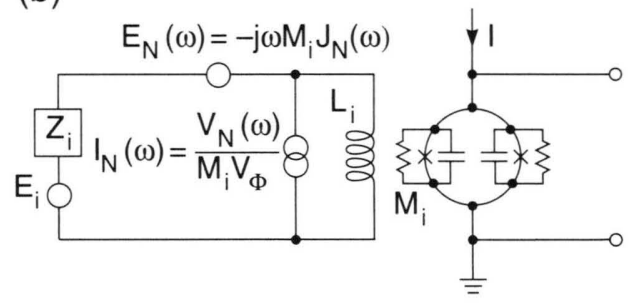

Fig. 3. (a) Noise sources in the dc SQUID. (b) Equivalent noise sources in a circuit coupled to the SQUID.

To use the SQUID as a voltage amplifier, one couples a superconducting coil of inductance $L_{\mathrm{i}}$ to it as shown in Fig. 3 b; the voltage source has an amplitude $E_{\mathrm{i}}(\omega)$ and an impedance $Z_{\mathrm{i}}(\omega)$. The voltage noise $V_{\mathrm{N}}(\omega)$ is equivalent to a flux noise $V_{N}(\omega) / V_{\Phi}$ in the SQUID and hence to an equivalent current noise source

$$
I_{\mathrm{N}}(\omega)=V_{\mathrm{N}}(\omega) / M_{\mathrm{i}} V_{\Phi}
$$

across the input coil, where $M_{\mathrm{i}}=\alpha\left(L L_{\mathrm{i}}\right)^{1 / 2}$ is the mutual inductance between the input coil and the SQUID. Similarly, $J_{\mathrm{N}}(\omega)$ is represented by a voltage source

$$
E_{\mathrm{N}}(\omega)=-j \omega M_{\mathrm{i}} J_{\mathrm{N}}(\omega)
$$

in series with $L_{\mathrm{i}}(j=\sqrt{-1})$. Thus, the SQUID amplifier, like any other amplifier, has two noise sources, $E_{\mathrm{N}}(\omega)$ and $J_{\mathrm{N}}(\omega)$. One can optimize the parameters of the input circuit to obtain the minimum noise temperature of the amplifier. This procedure is complicated by the fact that the SQUID is loaded by the input circuit and also reflects an additional impedance into the input circuit [20-22]. For our present purpose, we ignore these details, neglect the correlation between $J_{\mathbf{N}}(t)$ and $V_{\mathbf{N}}(t)$ and assume that $\alpha=1$ to give a simplified, approximate treatment that, nonetheless, yields results that are correct to within factors of 2 .

To begin with, we assume that the source impedance is a resistor, and set $Z_{\mathrm{i}}=R_{\mathrm{i}}$. In the standard treatment of the noise temperature of amplifiers [23], the optimum source resistance is found to be $E_{\mathrm{N}}(f) /$ $I_{\mathrm{N}}(f)$. Accordingly, with the aid of (2.1), (2.2), (2.3), (2.5), and (2.6) we find an optimized source resistance

$$
R_{\mathrm{i}}^{\mathrm{opt}} \approx \omega L_{\mathrm{i}} .
$$

Further, the optimized noise temperature is $\left[S_{\mathrm{E}}(f) S_{\mathrm{I}}(f)\right]^{1 / 2} / 2 k_{\mathrm{B}}$, where $S_{\mathrm{E}}(f)$ and $S_{\mathrm{I}}(f)$ are the spectral densities of $E_{\mathrm{N}}(t)$ and $I_{\mathrm{N}}(t)$, respectively. Using Eqs. (2.2), (2.3), (2.5) and (2.6), we find

$$
T_{\mathrm{N}}^{\mathrm{opt}} \approx 40 f T / V_{\Phi} .
$$

We note that $T$ increases linearly with both the operating frequency $\omega$ and the bath temperature $T$. Since $V_{\Phi}$ is a frequency of typically $25 \mathrm{GHz}$, for signal frequencies of $1 \mathrm{MHz}$ or less the noise temperature will be far below the bath temperature.

Finally, we estimate the power gain. From Fig. 3 b, we see that an input voltage $E_{\mathrm{i}}$ generates a flux $M_{\mathrm{i}} E_{\mathrm{i}} /$ $\left(R_{\mathrm{i}}+j \omega L_{\mathrm{i}}\right)$ in the SQUID and hence an output voltage $E_{0}=M_{\mathrm{i}} E_{\mathrm{i}} V_{\Phi} /\left(R_{\mathrm{i}}+j \omega L_{\mathrm{i}}\right)$. Assuming a SQUID dynamic resistance $R$ at the operating point and using (2.7), we find a power gain

$$
G \approx\left|E_{0}\right|^{2} R_{\mathrm{i}} /\left|E_{\mathrm{i}}\right|^{2} R \approx V_{\Phi} / 4 \omega .
$$

Thus, for $V_{\Phi}=25 \mathrm{GHz}$ and a signal frequency of $1 \mathrm{MHz}$, we find a gain of about $30 \mathrm{~dB}$.

Figure 4 shows the frequency dependence of $T_{\mathrm{N}}$ extrapolated from the measured value of a particular amplifier [22] operated at $100 \mathrm{MHz}$. The performance achieved is somewhat poorer than that predicted by (2.8), in part because the more complete treatment gives a somewhat higher numerical prefactor. In addition, in a real amplifier, the presence of parasitic inductance and capacitance and the fact that $\alpha<1$ degrade the performance. It should be emphasized that the noise temperature relates only to the SQUID and its input coil, and does not include Nyquist noise generated in the source resistance $R_{\mathrm{i}}$. Thus, the total system noise temperature is $T_{\mathrm{N}}+T$, and greatly exceeds $T_{\mathrm{N}}$ at low frequencies. The noise temperature shown in Fig. 4 is two orders of magnitude higher than that for an ideal, quantum limited amplifier, $T_{\mathrm{N}}^{\mathrm{Q}}=h f / k_{\mathrm{B}} \ln 2$. Modest improvements in $T_{\mathrm{N}}$, say by a factor of 3 to 5 , could be made by reducing the junction capacitance, enabling one to increase the shunting resistance [16], and by decreasing the loop inductance [18]. The upper curve in Fig. 4 represents typical values of semiconductor amplifiers; particularly at high frequencies, further improvements can be achieved by cooling the 


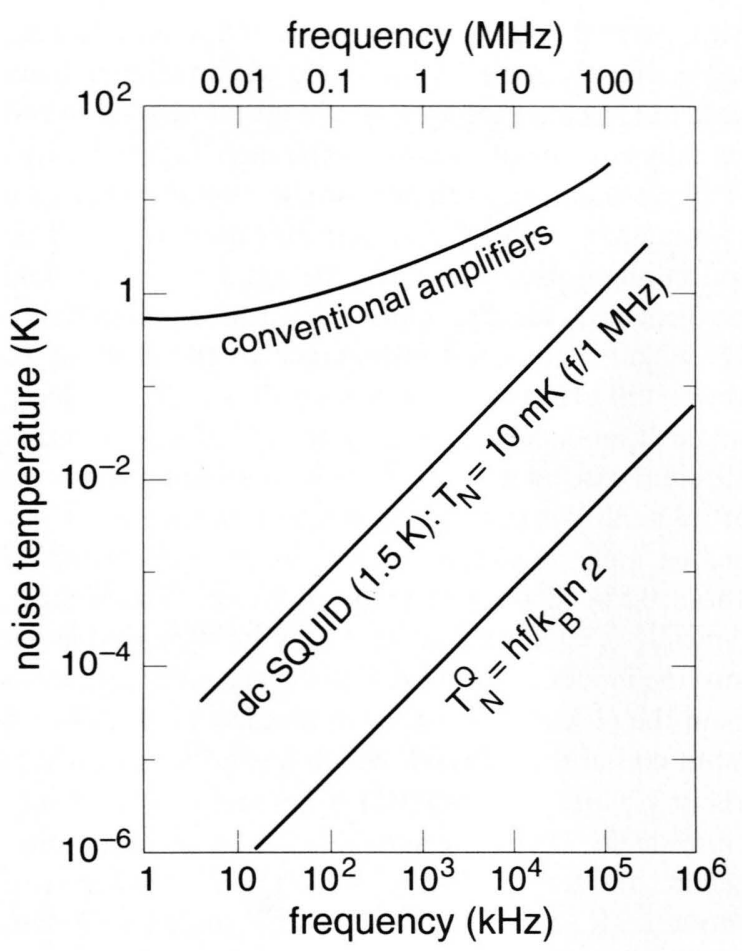

Fig. 4. Noise temperature vs. frequency for a dc SQUID at $1.5 \mathrm{~K}$, an ideal quantum limited amplifier, and typical conventional amplifiers at room temperature.

front stage of the amplifier, for example, a GaAs FET. We see that at the lower frequencies the noise temperature of the SQUID amplifier is very much smaller than that of conventional amplifiers, and it is this frequency range is relevant to our spectrometer.

To complete this section, we observe that in our magnetic resonance experiments we replace the voltage source in series with a resistor with a pickup coil of inductance $L_{\mathrm{p}}$ that surrounds the precessing nuclear spins. We choose $\omega L_{\mathrm{p}} \approx R_{\mathrm{i}}$ to achieve the optimization condition $L_{\mathrm{p}} \approx L_{\mathrm{i}}[10]$. The precessing spins induce a rate of change of flux $-j \omega \Phi$ in $L_{\mathrm{p}}$, where $\Phi$ is the flux due to the spins threading the pickup loop. Since the input circuit is entirely superconducting with a series impedance $j \omega\left(L_{\mathrm{i}}+L_{\mathrm{p}}\right)$, the current induced is $-\Phi /\left(L_{\mathrm{i}}+L_{\mathrm{p}}\right)$; this is simply the current required at any instant of time to maintain flux quantization in the input circuit. Thus, the amplifier now responds to $\Phi$ rather than $\mathrm{d} \Phi / \mathrm{d} t$. The fact that the input circuit is resistanceless implies that it generates no noise, enabling us to take full advantage of the low noise of the SQUID. By the same token, the lack of a

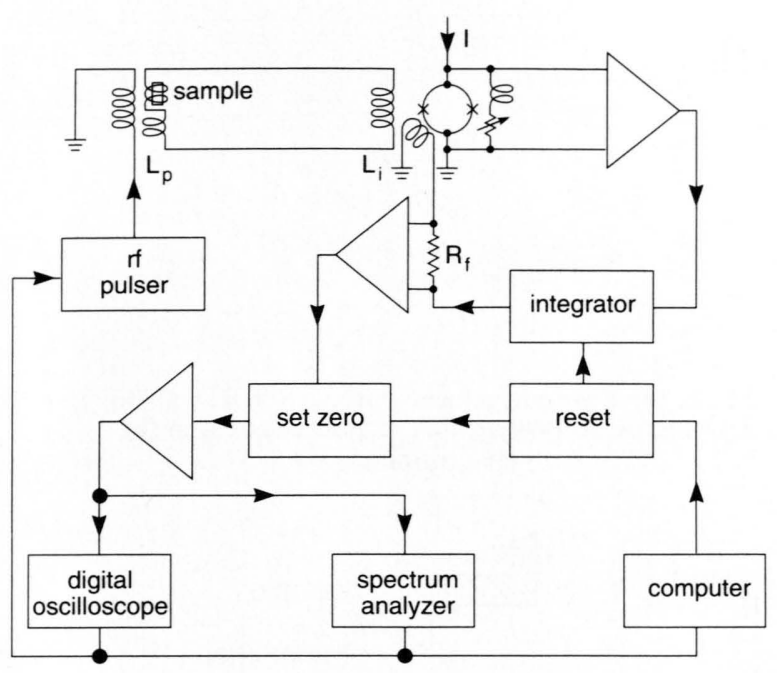

Fig. 5. Fourier transform spectrometer configured for nuclear quadrupolar resonance.

dissipative element implies that the concept of noise temperature is no longer strictly valid. However, the noise contribution of the SQUID is still given by (2.8) if one understands that it now represents a voltage noise spectral density $4 k_{\mathrm{B}} T_{\mathrm{N}} \omega L_{\mathrm{i}}$ in the input circuit.

\section{SQUID NQR Spectrometer}

The spectrometer consists of three main components: a flux-locked SQUID, a sample cell, and a computer-controlled system for supplying pulses to the sample and recording the response. We briefly describe each in turn.

The original version of our spectrometer involved a $500 \mathrm{kHz}$ flux modulation of the SQUID, and its bandwidth was limited to about $200 \mathrm{kHz}$. The current version, shown in Fig. 5, uses a direct-coupled readout scheme known as addition positive feedback (APF) [24] that is simpler and extends the bandwidth to above $1 \mathrm{MHz}$ [15]. In the APF scheme (Fig. 6a), a coil in series with a variable resistor, actually a cooled MESFET, is connected in parallel with the current-biased SQUID. When a magnetic flux is applied to the SQUID, the resultant voltage change causes a current flow through the coil which in turn induces an additional flux into the SQUID. Depending on the sign of $V_{\Phi}$, this flux either supports the applied flux, producing positive feedback or opposes it, producing negative feedback. The resultant $V-\Phi$ curve becomes 


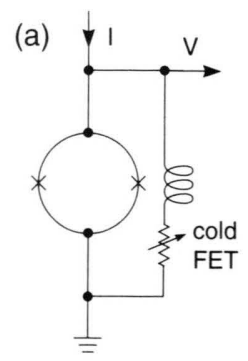

(b)

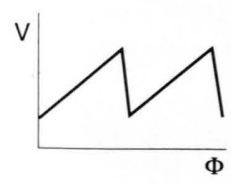

Fig. 6. (a) Readout scheme for dc SQUID giving rise to asymmetric voltage vs. flux response shown in (b).

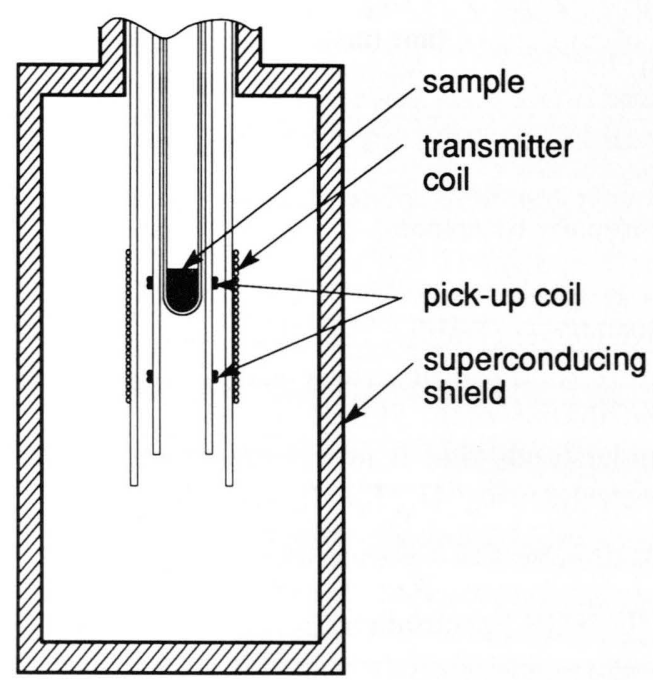

Fig. 7. Sample cell.

asymmetric, the steeper slope corresponding to positive feedback. One controls the degree of feedback by adjusting the resistance of the MESFET. The enhanced value of $V_{\Phi}$ on the steeper slope, typically $200 \mu \mathrm{V} / \Phi_{0}$ or more, enables one to connect the SQUID directly to a room temperature preamplifier, which has a typical input voltage noise of $0.5 \mathrm{nV}$ $\mathrm{Hz}^{-1 / 2}$, without sacrificing resolution. After amplification, the signal passes through a single-pole integrator and then, via a feedback resistor $R_{\mathrm{F}}$, through a coil inductively coupled to the SQUID. This flux-locked loop maintains the SQUID near the midpoint of the steep part of the $V-\Phi$ curve. The voltage developed across $R_{\mathrm{F}}$ is proportional to the flux in the SQUID. Typically, the flux noise is $2 \mu \Phi_{0} \mathrm{~Hz}^{-1 / 2}$ and the frequency response $(3 \mathrm{~dB})$ is $0-1 \mathrm{MHz}$.

The sample and the transmitter and receiver coils are enclosed in a cylindrical superconducting shield with internal length and diameter of 130 and $30 \mathrm{~mm}$, respectively [Figure 7]. The shield was machined from lead and has a wall thickness of $3 \mathrm{~mm}$. The pickup coil actually consists of a pair of coils, each with two turns of $75 \mu \mathrm{m}$-diameter niobium wire, wound in opposition $15 \mathrm{~mm}$ apart on a $8.5 \mathrm{~mm}$ diameter quartz tube. This coil configuration measures the axial magnetic field gradient and, ideally, is insensitive to uniform fields. We estimate the total inductance of the coils to be about $150 \mathrm{nH}$. The transmitter coil is a $30 \mathrm{~mm}$-long, single layer solenoid wound from $225 \mu$ m-diameter niobium wire on a quartz tube with an outer diameter of $14 \mathrm{~mm}$. We adjust the relative positions of the pickup and transmitter coils to achieve a balance of about $0.5 \%$ in the pickup gradiometer. The sample, typically $0.5 \mathrm{~g}$, is packed into a pyrex tube that slides into the inner quartz tube. The superconducting leads from the pickup coils are wire-bonded to the 20-turn input coil of the SQUID, which has an inductance of about $120 \mathrm{nH}$. The SQUID is surrounded by a niobium shield. The entire assembly is immersed in liquid helium, the temperature of which can be lowered to about $1.5 \mathrm{~K}$, and the dewar is surrounded with two concentric mu-metal shields to reduce the earth's magnetic field and magnetic noise. The sample tube can be inserted into and removed from the spectrometer via an o-ring seal at the top of the cryostat.

We now describe the operation of the spectrometer. Triggered by the computer, a function generator produces a sinusoidal signal containing between 1 and 30 cycles, starting at zero phase. The pulse is amplified and coupled to the transmitter coil via a low pass filter and four stages of crossed diodes. Theses diodes present a high impedance to the transmitter coil when the pulse is turned off, thus minimizing the noise coupled into the SQUID during the measurement. The precessing spins induce a flux in the pickup coil which is amplified by the SQUID. Despite the attenuation afforded by the gradiometer configuration, the initial $\mathrm{rf}$ pulse coupled into the SQUID would drive the integrator into saturation, from which it would take a long time to recover after the pulse was turned off. We overcome this problem by shorting the integrator capacitor with a photo-coupled analog switch during the pulse, and opening this switch after the pulse is removed. After the operation of the flux-locked loop has been restored, the output in general contains an offset which is removed by a set-zero circuit. The dead time between the removal of the rf pulse varies from about $15 \mu$ s for a $0.1 \mathrm{mT}$ peak-to-peak pulse to about 
$80 \mu$ s for $10 \mathrm{mT}$ peak-to-peak pulse. The amplified signal is connected to a spectrum analyzer and a digital oscillator, both of which store their data in the computer.

\section{4. $\mathrm{NQR}$ in $\mathrm{NH}_{4} \mathrm{ClO}_{4}$}

As an example of the application of our NQR spectrometer, we describe our studies of the zero field resonance in ammonium perchlorate $\left(\mathrm{NH}_{4} \mathrm{ClO}_{4}\right)$ at liquid helium temperature. Neutron diffraction [25] reveals an orthorhombic crystal structure in which the nitrogen and chlorine atoms lie on planes with mirror symmetry. The ammonium group has a low energy for reorientation via quantum tunneling of the four protons [26]; as a result the librational ground state levels are split. The spatial and spin wave function of each of these levels is characterized by one of the three irreducible representations of the tetrahedral group $T$, namely $A, E$ or $T$.

To obtain the results [13] presented here, we used the earlier version of our spectrometer with a flux modulated SQUID $[12,14]$. The zero-field spectrum of $0.5 \mathrm{~g}$ of powdered $\mathrm{NH}_{4} \mathrm{ClO}_{4}$ excited by a two-pulse sequence is shown in Figure $8 \mathrm{a}$. Each pulse was a single cycle at $45 \mathrm{kHz}$, the first with a peak-to-peak amplitude of $4 \mathrm{mT}$ followed $4 \mathrm{~ms}$ later by the second with twice the amplitude. The spectrum, averaged over 16,000 pulse sequences with a recycle rate of $3 \mathrm{~Hz}$, shows a spin echo $4 \mathrm{~ms}$ after the second pulse. The signal has been demodulated at $35 \mathrm{kHz}$. Figure $8 \mathrm{~b}$ shows the Fourier transform of the echo, consisting of three sharp peaks at $17.4,38.8$ and $56.2 \mathrm{kHz}$. The middle peak is the strongest in this figure because it is closest to the excitation frequency. The frequencies of the two lower resonances add up to the highest frequency, indicating that we are exciting and observing simultaneously the transition of a three-level system. To our knowledge, the $17.4 \mathrm{kHz}$ resonance is the lowest NQR frequency ever detected directly. The peak at $10.6 \mathrm{kHz}$ is due to a magnetoacoustic resonance of the input circuit.

To confirm that the resonant peaks arose from the ${ }^{14} \mathrm{~N}$ quadrupole interaction we performed several subsidiary experiments. First, when we enriched the $\mathrm{NH}_{4} \mathrm{ClO}_{3}$ with ${ }^{15} \mathrm{~N}$, which has no quadrupole moment, we observed no signal. Second, we recorded the amplitude of the free induction decay (FID) following the application of a soft pulse of known variable am-
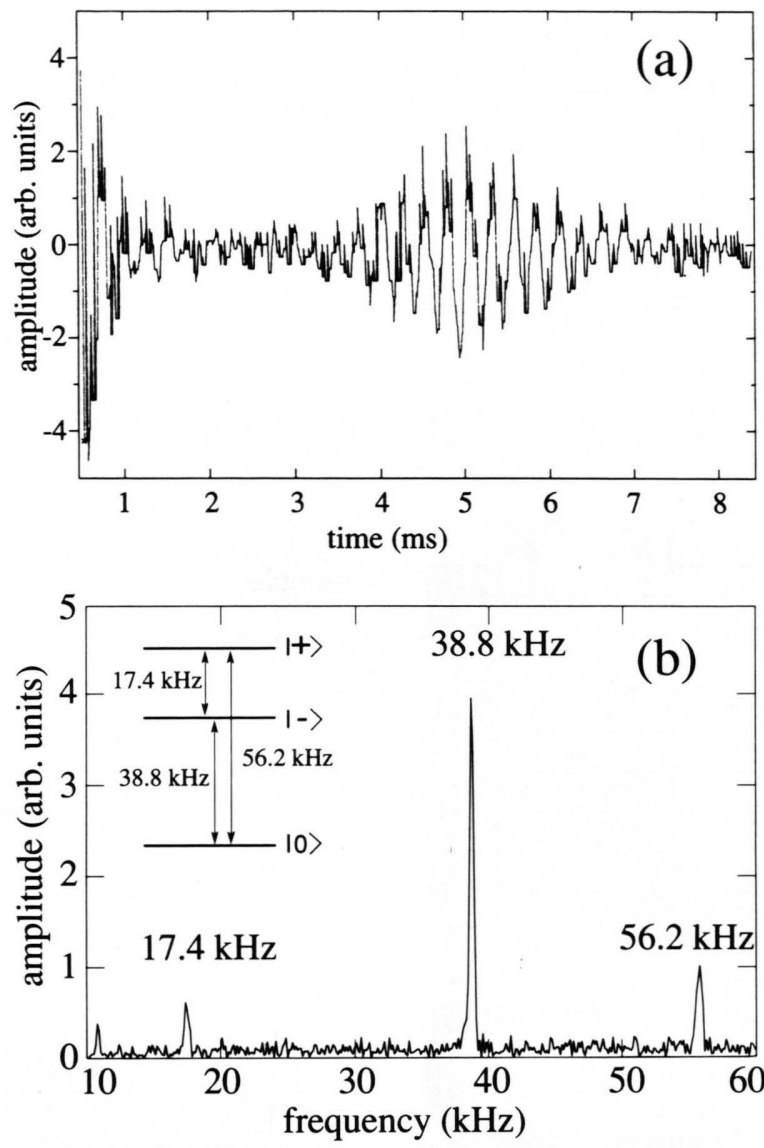

Fig. 8. (a) Spin echo observed at $1.5 \mathrm{~K}$ from ${ }^{14} \mathrm{~N}$ nuclei in $\mathrm{NH}_{4} \mathrm{ClO}_{4} 4 \mathrm{~ms}$ after second pulse. Real time signal has been demodulated at $35 \mathrm{kHz}$ for purpose of display. (b) Fourier transform of spin echo showing three resonant peaks due to transitions between energy levels (inset) of ${ }^{14} \mathrm{~N}$ nuclei in the presence of electric field gradient of $\mathrm{NH}_{4} \mathrm{ClO}_{4}$. In inset, $|0\rangle=|1,0\rangle$ and $| \pm\rangle=(|1,1\rangle \pm|1,-1\rangle) / \sqrt{2}$, where $|I, m\rangle$ is the eigenstate of the ${ }^{14} \mathrm{~N}$ nucleus with $I=1$ and $I_{z}=m$.

plitude that excited only the $38.8 \mathrm{kHz}$ transition. As we see in Fig. 9, the measured signal amplitude A could be fitted with the expression

$A(\omega \tau)=[\cos \omega \tau-(\sin \omega \tau) / \omega \tau] / \omega \tau$,

where $\omega=\gamma B_{1}, B_{1}$, and $\tau$ are the peak-to-peak amplitude and duration of the pulse and $\gamma=(1.9 \pm 0.2)$ $\times 10^{7} \mathrm{~Hz} /$ tesla is the fitted gyromagnetic ratio. Equation (4.1) is the expected dependence of the FID amplitude on the tipping angle $\gamma B_{1} \tau$ for a spin-1 nucleus in an asymmetric field gradient [4], and includes an angular average over the random orientation of the crystals in the powdered sample. The fitted value for $\gamma$ is in good agreement with the known value for ${ }^{14} \mathrm{~N}$, 


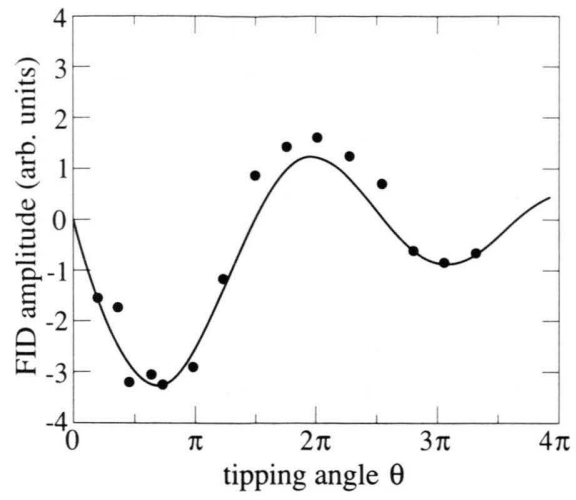

Fig. 9. FID amplitude vs. tipping angle $\theta=\omega \tau$. Solid circles are experimental data, curve is a fit to (4.1).

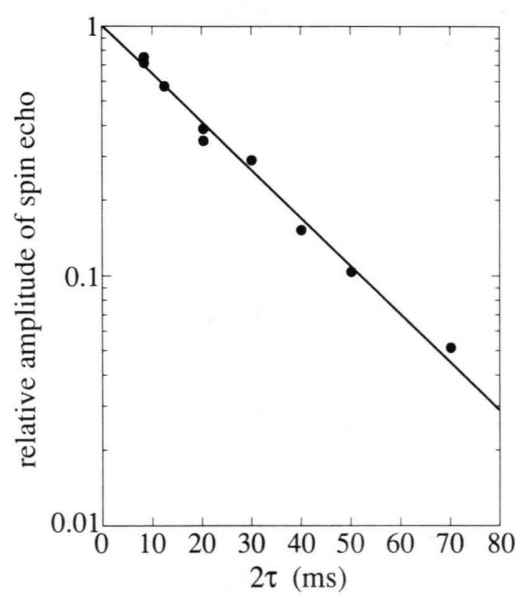

Fig. 10. Spin-echo amplitude vs. $2 \tau$ ( $\tau$ is delay time between pulses) for $38.8 \mathrm{kHz}$ transition at $1.5 \mathrm{~K}$. Solid circles are experimental data, solid line is fit to experimental decay with $T_{2}=22 \mathrm{~ms}$.

$\gamma=1.9325 \mathrm{~Hz} /$ tesla. Third, we performed a conventional pulsed high-field NMR experiment at $4.2 \mathrm{~K}$ in a magnetic field of $5.75 \mathrm{~T}$. The "powder pattern" spectrum showed splittings of $\pm\left(f_{2}-f_{1}\right) / 2, \pm\left(f_{1}+f_{3}\right) / 2$, and $\pm\left(f_{2}+f_{3}\right) / 2$, where $f_{1}, f_{2}, f_{3}$ are the resonant frequencies shown in Figure $8 \mathrm{~b}$. These are the frequencies expected for a spin-1 nucleus with a weak quadrupole interaction. Needless to say, the determination of the positions of the peaks in this experiment was much more uncertain than in our zero-field measurements.

From the resonant frequencies in Fig. $8 \mathrm{~b}$ we can obtain the ${ }^{14} \mathrm{~N}$ quadrupole coupling constant $e^{2} q Q / h$ and asymmetry parameter $\eta=\left(V_{x x}-V_{y y}\right) / V_{z z}$ at $1.5 \mathrm{~K}$.
Here $V_{x x}, V_{y y}$, and $V_{z z}$ are the electric field gradients along the principal axes, $q=V_{z z} / e$, and $Q$ is the quadrupole moment. For a spin 1 nucleus [4], the three energy levels are $-2 h v_{Q}, h v_{Q}(1-\eta)$ and $h v_{Q}(1+\eta)$, where $v_{Q}=e^{2} q Q / 4 h$, and the frequencies of the corresponding transitions among them are $2 h v_{Q} \eta, v_{Q}(3-\eta)$ and $v_{Q}(3+\eta)$. Inserting the measured frequencies we find $\eta=0.55$ and $e^{2} q Q /$ $h=63.3 \mathrm{kHz}$.

Finally, we measured the longitudinal and transverse relaxation times, $T_{1}$ and $T_{2}$. From a stimulated echo experiment [27] we found the unexpectedly short time $T_{1}=63 \pm 6 \mathrm{~ms}$ for the $38.8 \mathrm{kHz}$ transition at $1.5 \mathrm{~K}$. There are two likely mechanisms for this fast relaxation rate: quadrupolar relaxation, or dipolar interactions with the surrounding protons that induce rapid transitions among the $T$ levels. If we model the latter interaction in terms of fluctuating isotropic fields we predict $T_{2}=2 T_{1}=126 \pm 12 \mathrm{~ms}$. However, as we see in Fig. 10, the value of $T_{2}$ measured from the dependence of the spin echo amplitude on the time $\tau$ between pulses [27] is much shorter, $22 \pm 2 \mathrm{~ms}$. This result tends to rule out the dipolar interaction hypothesis. As a further confirmation, we measured the initial FID amplitude of partially deuterated $\mathrm{NH}_{4} \mathrm{ClO}_{4}$, maintaining the $3 \mathrm{~Hz}$ recycle rate. Now if the magnetic dipolar process occurred, we would expect it to induce rapid relaxation only in those ${ }^{14} \mathrm{~N}$ nuclei surrounded by protons in $T$ states; deuteration changes the symmetry and should strongly affect the fraction of ${ }^{14} \mathrm{~N}$ that can be magnetically relaxed by the protons. In fact, within the experimental uncertainty, we observed no change in the initial FID amplitude with deuteration. These two observations rule out dipolar interactions as a significant source of ${ }^{14} \mathrm{~N}$ relaxation, and indicate that fluctuating electric field gradients are the dominant mechanism.

\section{Concluding Remarks}

The low noise of our SQUID-based NQR spectrometer has enabled us to detect directly pulsed ${ }^{14} \mathrm{~N}$ quadrupole resonances at frequencies as low as $17.4 \mathrm{kHz}$. The fact that the system is broadband (in the latest version $0-1 \mathrm{MHz}$ ) rather than tuned allowed us to excite and observe all three transitions of the threelevel system simultaneously. Furthermore, using spin echoes we have made the first direct measurements of $T_{1}$ and $T_{2}$ in a polycrystalline solid at a frequency 
below $1 \mathrm{MHz}$. We note that Sager et al. [28] subsequently built a similar spectrometer, and also observed the NQR spectra of $\mathrm{NH}_{4} \mathrm{ClO}_{4}$. Although not reported here, in other experiments we detected ${ }^{2} \mathrm{D}$ NQR in perdeuterated toluene and perdeuterated picoline, and low-frequency NMR (at about $50 \mathrm{kHz}$ ) in ${ }^{195} \mathrm{Pt}$ and ${ }^{63} \mathrm{Cu} /{ }^{65} \mathrm{Cu}$ in a magnetic field of 6 mtesla [14].

One could improve the capability of our existing spectrometer by adding a variable temperature stage, although the signals would be correspondingly smaller at higher temperatures. This loss in signal amplitude could be overcome by averaging for longer times, by decreasing the noise temperature of the SQUID amplifier or, in appropriate cases, by prepolarizing the nuclei. The sensitivity, resolution and broad bandwidth of the SQUID based spectrometer make it very attractive for low-frequency NQR, NMR, and zero-field NMR studies of a wide range of solids, particularly in the polycrystalline or amorphous state. One can employ the many pulsed tech-

[1] M. Mehring, Principles of High Resolution NMR in Solids, Springer-Verlag, Berlin 1983, 2nd ed.

[2] C. P. Slichter, Principles of Magnetic Resonance, Springer-Verlag, Berlin 1983, 3rd ed.

[3] A. Pines, in Proceedings of the Bloch Symposium, edited by W. A. Little, World Scientific, Singapore 1990.

[4] T. P. Das, and E. L. Hahn, Solid State Physics, edited by F. Seitz and D. Turnbull, Academic Press, New York 1958, Suppl. 1.

[5] N. F. Ramsey, and R. V. Pound, Phys. Rev. 81, 278 (1951); A. G. Redfield, Phys. Rev. 130, 589 (1963); R. L. Strombotne and E. L. Hahn, Phys. Rev. 133, A1616 (1964); D. P. Weitekamp, A. Bielecki, D. Zax, K. Zilm, and A. Pines, Phys. Rev. Lett. 50, 1807 (1983).

[6] D. T. Edmonds, Phys. Rep. 29, 233 (1977); F. Noack, Progr. NMR Spectrosc. 18, 171 (1986).

[7] R. Tycko, Phys. Rev. Lett. 60, 2734 (1988).

[8] F. N. H. Robinson, J. Phys. E: Sci. Instrum. 15, 814 (1982).

[9] Donghoon Lee, S. J. Gravina, and P. J. Bray, Z. Naturforsch. 45 A, 268 (1990).

[10] See, for example, J. Clarke, SQUIDS: Theory and Practice, in The New Superconducting Electronics (Kluwer Academic, 1993) Ed. H. Weinstock and R. W. Ralston, p. 123.

[11] C. Connor, J. Chang, and A. Pines, Rev. Sci. Instr. 61, 1059 (1990).

[12] N. Q. Fan, and J. Clarke, Rev. Sci. Instrum. 62, 1453 (1991).

[13] M. D. Hürlimann, C. H. Pennington, N. Q. Fan, J. Clarke, A. Pines, and E. L. Hahn, Phys. Rev. Lett. 69, 684 (1992).

[14] Non Fan, Ph.D. thesis, University of California, Berkeley, California. niques developed for high-frequency NMR and NQR, including, for example, multidimensional spectroscopy.

\section{Acknowledgements}

I thank Non Fan who developed the first NQR spectrometer and obtained the first NMR and NQR spectra, Dinh Ton who developed the faster spectrometer described in this paper, and Martin Hürlimann, Charles Pennington, Erwin Hahn and Alex Pines for obtaining and interpreting the $\mathrm{NH}_{4} \mathrm{ClO}_{3}$ spectra. I am grateful to Dinh Ton and P. J. Bray for their thoughtful comments on the manuscript. This work was supported by the Director, Office of Energy Research, Office of Basic Energy Sciences, Materials Sciences Division of the U.S. Department of Energy under contract number DE-AC03-76SF00098, by the Swiss Nationalfonds, by IBM and by Quantum Design.

[15] Dinh Ton, and J. Clarke (unpublished).

[16] W. C. Stewart, Appl. Phys. Lett. 12, 277 (1968); D. E. McCumber, J. Appl. Phys. 39, 3113 (1968).

[17] M. B. Ketchen, and J. M. Jaycox, Appl. Phys. Lett. 40, 736 (1982).

[18] C. D. Tesche, and J. Clarke, J. Low Temp. Phys. 27, 301 (1977).

[19] C. D. Tesche, and J. Clarke, J. Low Temp. Phys. 37, 397 (1979).

[20] J. M. Martinis, and J. Clarke, J. Low Temp. Phys. 61, 227 (1985).

[21] C. Hilbert, and J. Clarke, J. Low Temp. Phys. 61, 237 (1985).

[22] C. Hilbert, and J. Clake, J. Low Temp. Phys. 61, 263 (1985).

[23] See, for example, F. N. H. Robinson, Noise and Fluctuations in Electronics Devices and Circuits, Clarendon Press, Oxford 1974, Chapter 12.

[24] D. Drung, R. Cantor, M. Peters, H. J. Scheer, and H. Koch, Appl. Phys. Lett. 57, 406 (1990).

[25] H. J. Prask, C. S. Choi, N. J. Chesser, and G. J. Rosasco, J. Chem. Phys. 88, 5106 (1988).

[26] W. Güttler, and J. U. von Schütz, Chem. Phys. Lett. 20, 133 (1973); J. W. Riehl, R. Wang, and H. W. Bernard, J. Chem. Phys. 58, 508 (1973); H. J. Prask, S. F. Trevino, and J. J. Rush, J. Chem. Phys. 62, 4556 (1975); R. F. Code, J. Higinbotham, and A. R. Sharp, Can J. Phys. 54, 239 (1976); J. Svare, J. Phys. C10, 4137 (1977); R. J. C. Brown, R. D. Weir, and E. F. Westrum, J. Chem. Phys. 91, 399 (1989).

[27] E. L. Hahn, Phys. Rev. 80, 580 (1950).

[28] R. E. Sager, A. D. Hibbs, D. N. Shykind, and B. T. Thorson, The NQR Newsletter 1, 15 (1993). 